\title{
Análise da segurança do uso de imunossupressores por pacientes insuficientes renais
}

\author{
Safety analysis of the immunosuppressants use in renal failure patients
}

Recebido em: 23/01/2020

Aceito em: $\quad 27 / 08 / 2020$
Fernanda TOALDO; Flavia Lorenzen dos SANTOS; Fernanda Cristina Ostrovski SALES; Jaqueline de Jesus ASCHENBRENNER Pontifícia Universidade Católica do Paraná - PUCPR. Rua Imaculada Conceição, 1155, Prado Velho, CEP 80215-901. Curitiba, PR, Brasil.

E-mail:flah.lorenzen@hotmail.com

\section{ABSTRACT}

Acute renal failure (ARF) and chronic renal failure (CRF) are pathologies with increasing incidence recently in Brazil. According to the Brazilian Society of Nephrology (2018), the number of patients with these comorbidities has increased by approximately $200 \%$ in 16 years, with organ transplantation as an alternative treatment. One of the main post-renal transplant therapies is the use of immunosuppressants to contain graft rejection. However, the safety of this pharmacotherapy is still discussed, due to the high probability of adverse reactions occurring. This study presented and analyzed numerical data regarding the occurrence of adverse drug reaction (ADR) related to immunosuppressants used as pharmacotherapy by patients with ARF or CRF hospitalized in a University Hospital of Curitiba county, PR. It is a retrospective observational study based on the analysis of physical and electronic records of the evolution of acute or chronic renal insufficiency patients through active search in immunosuppressive dispensing tables. This study included records of 162 patients, and 118 of them had one or more possible immunosuppressive reactions.

We observed that the most prevalent ADR in the study was "opportunistic infection" and the lowest prevalence "hypoalbuminemia." For better result reliability, Naranjo's algorithm was applied, where "infection" remained relevant in $26 \%$ of its cases classified by the algorithm as "probable." Given the results, the importance of the clinical pharmacist in the optimization of the immunosuppressive pharmacotherapy used by the renal insufficient patients of the hospital in question is observed.

Keywords: clinical pharmacy; immunosuppressants; adverse drug reactions.

\section{RESUMO}

A insuficiência renal aguda (IRA) e a insuficiência renal crônica (IRC) consistem em patologias com incidência crescente ao longo dos últimos anos no Brasil. Segundo a Sociedade Brasileira de Nefrologia (2018), o número de pacientes com estas comorbidades tem aumentado cerca de $200 \%$ em 16 anos, tendo como alternativa de tratamento o transplante do órgão. Uma das principais terapias pós-transplante renal 
consiste no uso de imunossupressores, com o objetivo de conter a rejeição do enxerto. Em contrapartida, a segurança desta farmacoterapia ainda é bastante discutida, pela alta probabilidade de ocorrência de reações adversas. Esse estudo objetivou apresentar e analisar dados numéricos referentes à ocorrência de Reação Adversa a Medicamentos (RAM) relacionados a imunossupressores utilizados como farmacoterapia por pacientes com IRA ou IRC, internados em um Hospital Universitário do Município de Curitiba. PR. Trata-se de um estudo retrospectivo observacional, baseado na análise de prontuários físicos e eletrônicos de pacientes insuficientes renais agudos ou crônicos, por meio de busca ativa em tabelas de dispensação de imunossupressores. Este trabalho incluiu prontuários de 162 pacientes, sendo que 118 destes apresentaram uma ou mais possíveis reações adversas de imunossupressores. A RAM de maior prevalência no estudo foi "infecção oportunista" e a de menor prevalência "hipoalbuminemia". Para maior confiabilidade dos resultados foi aplicado Algoritmo de Naranjo, onde "infecção" permaneceu relevante em 26\% dos seus casos classificada pelo algoritmo como "provável". Diante dos resultados expostos, observa-se a importância do farmacêutico clínico na otimização da farmacoterapia imunossupressora utilizada por pacientes insuficientes renais do hospital em questão.

Palavras-chave: Farmácia Clínica; imunossupressores; Reações Adversas a Medicamentos.

\section{INTRODUÇÃO}

A insuficiência renal pode ser definida como a incapacidade dos rins de filtrar o sangue, eliminando resultados de metabolismo, como ureia e/ ou creatinina, por exemplo, que podem ficar acumuladas no organismo quando os rins não estão funcionando bem. Tal alteração funcional dos rins pode ser classificada (conforme suas características, reversibilidade e desfecho) como aguda ou crônica (1). A Insuficiência Renal Aguda (IRA) é descrita por uma redução da função renal que se mantém por períodos variáveis, resultando na incapacidade dos rins em realizarem suas funções básicas e a manutenção da homeostase (2). A Insuficiência Renal Crônica (IRC) é a fase mais avançada da doença renal, com intensidade agressiva da capacidade compensadora do rim, com comprometimento lento e caráter de irreversibilidade, comprometendo de forma vagarosa praticamente todas as funções renais, tanto as urinárias como endócrinas (3).

Estima-se que o número de diálises realizadas no Brasil vem crescendo ao longo dos anos. Em 2000, a estimativa era de 42.695 e, em 2016, esse número chegou a 122.825 , o que representa um aumento de cerca de $200 \%$. O último Censo da
Sociedade Brasileira de Nefrologia (SBN) mostrou que o número de novos casos de pacientes insuficientes renais é de aproximadamente 40.000 pacientes ao ano, tendo um aumento gradativo em pacientes que realizam transplante renal, chegando cerca de 5.900 pacientes ao ano (4).

$\mathrm{Na}$ IRC, os imunossupressores constituem a farmacoterapia utilizada para prevenir e/ou tratar rejeição de transplantes renais. Existem quatro classes principais de imunossupressores: glicocorticoides, inibidores de calcineurina, fármacos antiproliferativos e antimetabólicos, e os anticorpos. Eles reduzem ou impedem reações imunológicas e manifestações ocorridas após o transplante. Comumente são administrados corticosteroides em associação com inibidores de calcineurina (ciclosporina ou tacrolimus), ou com fármacos antiproliferativos (azatioprina ou micofenolato de mofetila) (5).

O objetivo, em um primeiro momento, é evitar a rejeição do órgão transplantado por completo e depois, inibir receptores celulares específicos para que possa ser reduzida a terapia imunossupressora em doses não tóxicas. Uma das principais preocupações com a farmacoterapia de imunossupressores são as reações adversas que eles podem causar no paciente (6). Em geral, as principais reações destes medicamentos apontadas pela literatura e 
estudos semelhantes são: nefrotoxicidade, distúrbios gastrintestinais e metabólicos, anemia, leucopenia, erupções cutâneas, complicações infecciosas, entre outros $(5,7,8)$.

Relacionando cada imunossupressor com suas respectivas reações adversas mais comumente notificadas, o tacrolimus é associado com nefrotoxicidade, neurotoxicidade, diabetes mellitus, hipertensão e distúrbios gastrointestinais; o sirolimus associado com hiperlipidemia e supressão da medula óssea; o micofenolato de mofetila, com a supressão da medula óssea e os sintomas gastrointestinais como os principais efeitos colaterais; e a azatioprina associada a supressão da medula óssea, distúrbios gastrointestinais e hepatotoxicidade $(9,10)$.

Com foco na melhoria da qualidade de vida do paciente, a prática da Farmácia Clínica possui a responsabilidade de prevenção e monitoramento de efeitos adversos, contribuições e intervenções na prescrição médica, melhorando assim a farmacoterapia do paciente, resultando em minimização de riscos e custos (11). Diante do exposto, pode ser destacada a importância de estudos que forneçam números relacionados à segurança da farmacoterapia imunossupressora frente às suas principais reações adversas, a fim de que os resultados destes auxiliem na previsão de manejo dos pacientes portadores de IRA ou IRC, usuários de imunossupressores. Assim, o presente estudo tem como objetivo apresentar a análise de dados referentes à ocorrência de reação adversa a medicamentos (RAM) relacionadas a imunossupressores utilizados como farmacoterapia por pacientes com IRA ou IRC, internados em um Hospital Universitário do Município de Curitiba, PR. Esse trabalho busca enfatizar também a importância da atuação de um farmacêutico clínico no hospital em estudo, principalmente em áreas críticas e de alto custo, onde as prescrições e procedimentos demandam maior atenção.

\section{MATERIAL E MÉTODO}

Por meio da revisão de literatura sobre os imunossupressores em pacientes insuficientes re- nais e suas principais reações adversas, foram definidas as seguintes palavras-chave: aplasia medular, infecções, dislipidemia, diabetes descompensado e distúrbios do trato gastrointestinal. Tais palavras-chave auxiliaram na busca dos prontuários dos pacientes a serem incluídos no presente estudo. Entretanto, no decorrer das análises foram acrescentadas outras reações adversas relatadas com frequência nos prontuários: desequilíbrio eletrolítico, perda de peso e apetite, úlceras/aftas orais, acidose metabólica, ataxia, parestesia e hipoalbuminemia. O projeto foi aprovado no Comitê de Ética em Pesquisa em Humanos da PUCPR (CAAE 72098817.0.0000. 0020).

Os critérios de inclusão utilizados para a seleção dos prontuários foram pacientes maiores de 18 anos, insuficientes renais agudos ou crônicos, em tratamento com os imunossupressores tacrolimus, micofenolato de sódio e azatioprina, padronizados pelo hospital em questão. A maioria dos pacientes que tiveram seus prontuários analisados pelo presente estudo, não estava internada para a operação de transplante nem o tinha feito recentemente. Os pacientes estavam internados devido a outros fatores e complicações de saúde. Foram coletados dados referentes a um período de 6 meses (setembro de 2017 a fevereiro de 2018), por meio de busca ativa em tabelas de dispensação de imunossupressores e prontuários físicos e eletrônicos de evolução dos pacientes insuficientes renais agudos e crônicos. Dados referentes à idade, gênero e sinergismo dos imunossupressores foram coletados através dos prontuários e tabulados para análise estatística simples.

Posteriormente à coleta de dados, as RAM foram classificadas quanto à causalidade segundo o Algoritmo de Naranjo (12) (Tabelas 1 e 2), que é o algoritmo mais consolidado para a busca de informações sobre as RAM, classificando-as em categorias: definida, provável, possível ou duvidosa. Quanto mais baixa a pontuação, mais duvidosa a reação adversa, enquanto uma alta pontuação converge para a definição da reação adversa como causa.

Os dados foram coletados e analisados quantitativamente, por análise de frequência. 
Tabela 1. Algoritmo de utilizado para determinação da causalidade de reações adversas a medicamentos (13).

\begin{tabular}{|c|c|c|c|c|}
\hline QUESTÕES & SIM & NÄ0 & DESCONHECIDO & SOMA/SCORES \\
\hline 1. Existem notificações conclusivas sobre esta reação? & +1 & 0 & 0 & \\
\hline 2. A reação apareceu após a administração do fármaco? & +2 & -1 & 0 & \\
\hline 3. A reação melhorou quando o fármaco foi suspenso? & +1 & 0 & 0 & \\
\hline 4. A reação reapareceu quando da sua readministração? & +2 & -1 & 0 & \\
\hline 5. Existem causas alternativas (até mesmo outro fármaco)? & -1 & +2 & 0 & \\
\hline 6. A reação reaparece com a introdução de um placebo? & -1 & +1 & 0 & \\
\hline 7. A concentração plasmática está em nível tóxico? & +1 & 0 & 0 & \\
\hline $\begin{array}{l}\text { 8. A reação aumentou com dose maior ou reduziu com } \\
\text { dose menor? }\end{array}$ & +1 & 0 & 0 & \\
\hline $\begin{array}{l}\text { 9. } 0 \text { paciente já experimentou semelhante reação } \\
\text { anteriormente com medicamentos de mesmo fármaco? }\end{array}$ & +1 & 0 & 0 & \\
\hline \multirow[t]{2}{*}{$\begin{array}{l}\text { 10. A reação foi confirmada por qualquer evidência } \\
\text { objetiva? }\end{array}$} & +1 & 0 & 0 & \\
\hline & & & TOTAL & \\
\hline
\end{tabular}

Fonte: Naranjo (12) apud Capucho (13).

Tabela 2. Somatório de scores proposto por Naranjo e cols. (1981), resultado da utilização do Algoritmo, a fim de determinar a causalidade de reações adversas a medicamentos.

\begin{tabular}{|c|c|}
\hline Somatório dos Scores & Classes de Causalidade \\
\hline 9 ou + & Definida \\
\hline 5 a 8 & Provável \\
\hline 1 a 4 & Possível \\
\hline 0 ou - & Duvidosa \\
\hline
\end{tabular}

Fonte: Naranjo (12) apud Capucho (13).

\section{RESULTADO E DISCUSSÃO}

Foram selecionados para o presente estudo, um total de 162 pacientes insuficientes renais com uso da terapia imunossupressora. Dentre eles, 118 (73\%) apresentavam uma ou mais RAM provenientes da farmacoterapia. Dos 118 pacientes considerados com RAM (100\%), a prevalência foi do sexo masculino, com 68 pacientes (58\%). Segundo um estudo acerca da predominância do sexo de pacientes em diálise ou hemodiálise e transplantados renais no Brasil, em sua maioria eram do sexo mas- culino, ultrapassando $50 \%$ do total dos pacientes em análise nos estudos comparativos (14).

Quanto à idade, como observado na Figura 1, a seguir, a maior prevalência das RAM ocorreu entre os 51 e 70 anos de idade, totalizando mais de $50 \%$, resultados que condizem com estudos comparativos (14). O baixo número de pacientes com RAM entre 71 e 80 anos de idade se justificou, pelo baixo número de pacientes com essa idade no estudo. Vale ressaltar que um dos motivos para tal seria a pequena incidência de transplantes nesta faixa etária, pois, segundo dados observados nos 
prontuários sobre o histórico prévio dos pacientes de maior faixa etária, os mesmos realizaram o transplante anteriormente. Tal achado converge com um estudo publicado em 2015, no qual a idade avançada foi considerada uma contraindicação para a cirurgia, devido aos seus altos riscos, levando assim os centros de transplantes renais em hesitar ao usar um recurso escasso como o enxerto renal num receptor com sobrevivência expectável limitada e preocupações relativamente à mortalidade e morbidade do procedimento neste grupo mais fragilizado (15).

Figura 1. Caracterização, quanto à faixa etária, dos pacientes de um hospital no Estado do Paraná, Brasil, com insuficiência renal que apresentaram Reações Adversas a Medicamentos (setembro de 2017 a fevereiro de 2018).

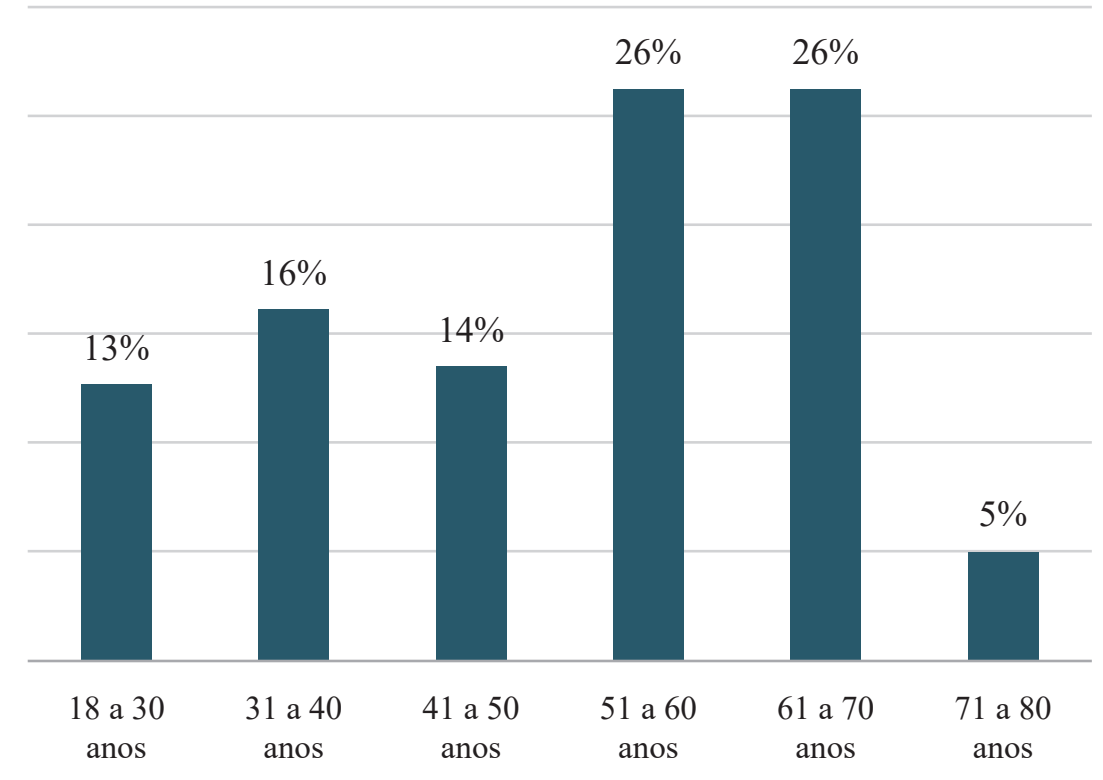

A Figura 2 traz informações sobre a distribuição das diferentes RAM encontradas no estudo. Infecção oportunista foi a reação de maior prevalência neste estudo, seguida de distúrbios do trato gastrointestinal (TGI): diarreia, náuseas, vômitos, constipação e dispepsia. Em muitos pacientes foi observado desequilíbrio eletrolítico, caracterizado pelos níveis baixos ou altos de alguns dos eletrólitos, perda de peso, falta de apetite e Diabetes Mellitus (DM) descompensado. A observação da depressão medular de algum tipo de célula sanguínea (eritrócitos, leucócitos e plaquetas) foi contemplada na RAM de aplasia medular. Dislipidemia, úlceras orais e aftas, acidose metabólica, ataxia, parestesia e hipoalbuminemia foram as RAM de menor prevalência no estudo.

Pacientes transplantados apresentam um alto risco de infecções oportunistas, visto que estão imunocomprometidos diante da terapia em questão, internados em um ambiente hospitalar e com regiões expostas onde o predomínio de infecções é elevado, como a incisão da cirurgia e vias aéreas. Nos primeiros meses pós-transplante ocorre uma prevalência de infecções causadas por bactérias, vírus e fungos, devido ao estado imunológico do paciente em conjunto ao ambiente hospitalar. Ao longo do tempo, a dosagem da terapia imunossupressora é ajustada a níveis não tóxicos, buscando o equilíbrio entre a máxima efetividade em evitar rejeição com a mínima supressão do sistema imunológico, o que faz o índice de infecções diminuir em comparação aos primeiros meses de terapia. Esta RAM é fortemente discutida na literatura e artigos científicos, sendo uma das mais relatadas, assim como ocorreu no presente estudo $(8,15,16,17)$. Esta reação é pertinente ao uso de todo os imunossupressores 
padronizados no hospital, sendo insignificante a diferença de sua predominância entre as classes (16).

Efeitos adversos no aparelho digestivo têm sido fortemente associados à terapia imunossupressora e descritos em literatura e estudos semelhantes $(9,17,18,19)$. Dentre esses, o micofenolato de mofetila comparado a azatioprina no transplante renal foi associado a um aumento de distúrbios do trato gastrointestinal (diarreia, vômito e dor abdominal). Podemos observar uma alta taxa de pacientes que apresentaram tal RAM. Entretanto este fato também pode ser correlacionado a outros fatores além dos imunossupressores, como outros fármacos administrados pelos pacientes, efeitos secundários a uma infecção, hábitos alimentares, entre outros (19).

Uma das principais funções dos rins é realizar o equilíbrio das concentrações de eletrólitos plasmáticos, mantendo assim a homeostase eletrolítica corporal. Quando ocorre a perda da função renal, essa atividade fica prejudicada, o que pode levar a um desequilíbrio, principalmente de sódio, potássio e cálcio (20). Este desequilíbrio foi altamente relatado pelos pacientes participantes do estudo e assim quantificado no gráfico acima. Todavia, esta reação pode estar correlacionada com a progressão da doença, visto que muitos pacientes pós-trans- plantados têm significativa melhora, mas não a sua cura total. Esta RAM é fracamente abordada pela literatura e artigos científicos de que seria decorrente de imunossupressores. Alguns estudos mencionam que em certas situações, como no caso da hipomagnesemia, está relacionada aos efeitos dos inibidores da calcinerina (tacrolimus) no transporte tubular. Porém pela inexistência de estudos que abordem esta reação aos imunossupressores, esta pode ser entendida como fator de confusão. (21)

Diabetes descompensado tem sido frequentemente relatado em estudos de pós-transplante como uma reação geralmente associada ao uso de imunossupressores, principalmente apontado ao uso unicamente do tacrolimus e/ou de sua combinação com o micofenolato de sódio. Alguns estudos semelhantes trazem dados interessantes, onde mostram que o tacrolimus tem maior risco de desenvolvimento desta complicação quando comparado aos demais imunossupressores. Isso porque estes fármacos podem inibir a síntese de insulina e reduzir sua excreção, como também podem causar uma resistência insulínica. Esta reação também pode ser decorrente da doença Diabetes Mellitus, comorbidade que muitos pacientes apresentaram, podendo estar descompensada pela má adesão ao tratamento da doença (22-23).

Figura 2. Distribuição de frequência das diferentes Reações Adversas a Medicamentos em pacientes com insuficiência renal de um hospital no Estado do Paraná, Brasil (setembro de 2017 a fevereiro de 2018).

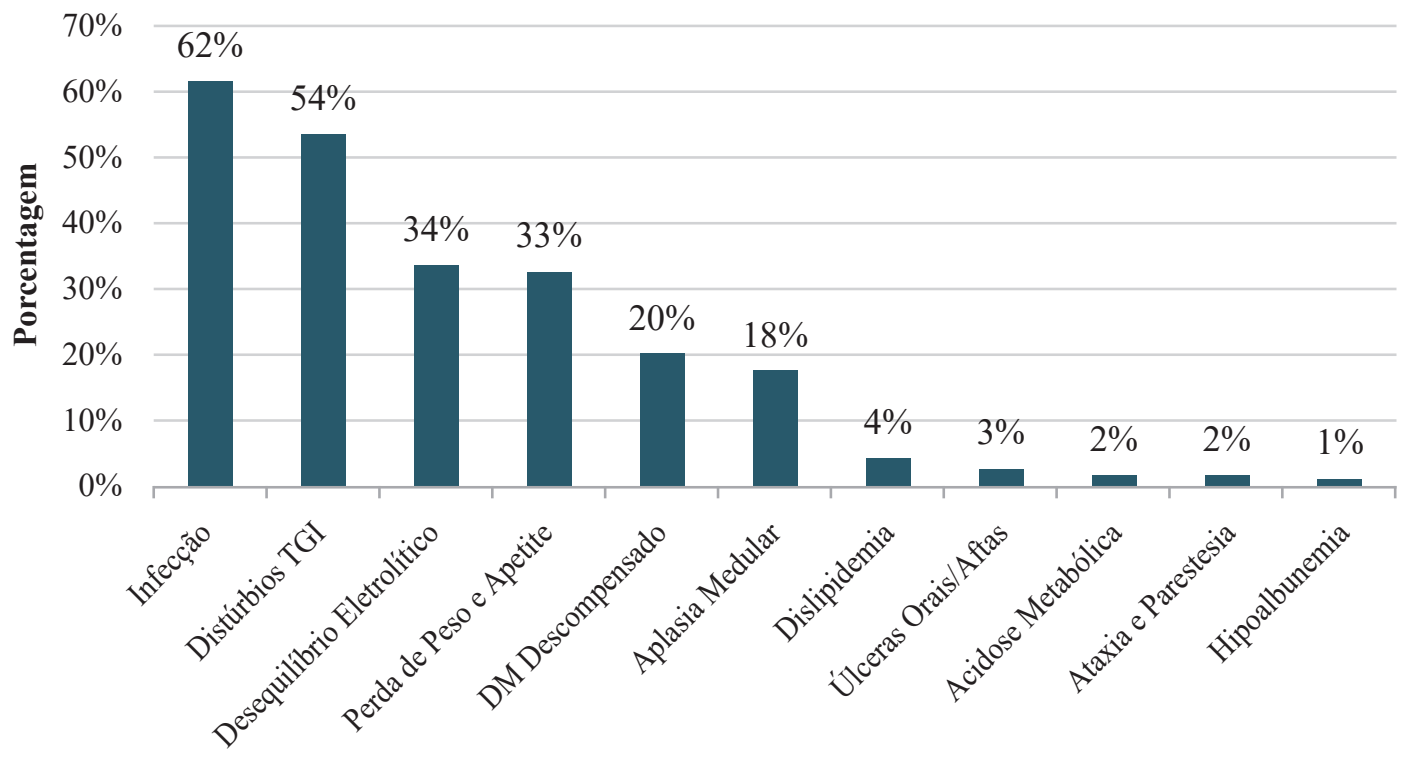


Em relação aos medicamentos imunossupressores utilizados, a azatioprina, pró-fármaco da 6-mercaptopurina, tem como mecanismo de ação a incorporação dos metabólitos, nucleotídeos 6-tioguanina, ao DNA e RNA, resultando em efeito citotóxico, além de estimularem mitocôndrias a levar à apoptose certas linhagens linfocitárias CD3 e CD28 (18). O micofenolato de sódio age na biossíntese das purinas, levando ao bloqueio da síntese de nucleotídeos de guanosina (GTP e dGTP), que são substratos para a síntese de DNA e RNA. Desta forma, ocorre inibição da síntese de ácido nucleico e consequentemente da proliferação celular. E o tacrolimus suprime a ativação das células $\mathrm{T}$ e a proliferação das células $\mathrm{B}$ induzida pelas células T-Helper, assim como a formação de linfocinas (como as interleucinas-2, -3 e o interferon $\gamma$ ) e a expressão dos receptores da interleucina-2 (24). Tais mecanismos de ação explicam a aplasia medular expressada na Figura 2. A principal linhagem sanguínea que apresentou depressão no presente estudo foram os leucócitos, com alta prevalência nos prontuários, fato justificado pela ação linfocitotóxica dos imunossupressores. Nem sempre essa reação foi relatada nos prontuários analisados; muitos apresentavam falta de informações, o que poderia estar relacionado ao baixo número de pa- cientes correlacionados nesta RAM. Estudos semelhantes apontam que aplasia medular é uma reação consideravelmente frequente em pacientes transplantados, principalmente nos primeiros meses, resultado não observado no presente estudo. O micofenolato de mofetila é o principal imunossupressor associado a reações adversas hematológicas, com exceção da leucopenia e da trombocitopenia, que são substancialmente maiores em pacientes com azatioprina $(17,23)$.

Em relação à dislipidemia, estudos relatam que essa reação é predominante em $60 \%$ dos pacientes pós-transplante. Entretanto, esta é uma reação multifatorial, que pode ser decorrente da doença pré-transplante, hábitos alimentares, perda da função do enxerto, entre outros (8). O baixo número de pacientes encontrado no presente estudo pode ser relacionado a, principalmente, dois fatores: apresentarem a patogenia pré-transplante ou o não relato da mesma durante a evolução hospitalar. Como descrito na literatura, o sirolimus é o imunossupressor mais comumente relacionado com risco de hiperlipidemia $(10,11)$.

A partir dos resultados foi aplicado o Algoritmo de Naranjo (Tabela 3), para confirmação da correlação entre as condições clínicas mencionadas

Tabela 3. Frequência de Reações Adversas a Medicamentos detectadas em pacientes com insuficiência renal de um hospital no Estado do Paraná, Brasil (setembro de 2017 a fevereiro de 2018), em relação à classificação de causalidade do Algoritmo de Naranjo.

\begin{tabular}{|l|c|c|c|c|}
\hline \multicolumn{1}{|c|}{ RAM } & Duvidosa & Possivel & Provável & Definida \\
\hline Infecção & $0 \%$ & $74 \%$ & $26 \%$ & $0 \%$ \\
\hline Distúrbios TGI & $42 \%$ & $55 \%$ & $3 \%$ & $0 \%$ \\
\hline Desequilíbrio Eletrolítico & $10 \%$ & $87,5 \%$ & $2,5 \%$ & $0 \%$ \\
\hline Perda de Peso e Apetite & $31 \%$ & $69 \%$ & $0 \%$ & $0 \%$ \\
\hline Aplasia Medular & $0 \%$ & $71 \%$ & $29 \%$ & $0 \%$ \\
\hline DM Descompensado & $12,5 \%$ & $87,5 \%$ & $0 \%$ & $0 \%$ \\
\hline Dislipidemia & $0 \%$ & $100 \%$ & $0 \%$ & $0 \%$ \\
\hline Acidose Metabólica & $50 \%$ & $50 \%$ & $0 \%$ & $0 \%$ \\
\hline Ataxia e Parestesia & $0 \%$ & $100 \%$ & $0 \%$ & $0 \%$ \\
\hline Úlceras Orais/Aftas & $0 \%$ & $100 \%$ & $0 \%$ & $0 \%$ \\
\hline Hipoalbuminemia & $0 \%$ & $100 \%$ & $0 \%$ & $0 \%$ \\
\hline
\end{tabular}

TGI: Trato Gastrointestinal; DM: Diabetes Mellitus. 
e a probabilidade de as mesmas serem RAM da farmacoterapia imunossupressora analisada. Foi obtida a classificação "Possível" para grande parte das RAM detectadas, fato que pode ser justificado por fatores que podem gerar incerteza na correlação entre o evento adverso e o uso do medicamento, como o uso de outros medicamentos ou associações medicamentosas; uma ou mais comorbidades no mesmo paciente e, por fim a própria evolução da insuficiência renal, não sendo possível obter uma resposta definida para a reação adversa. Como discutido anteriormente, os imunossupressores são fármacos que alteram a proliferação celular, resultando em uma baixa produção e/ou destruição das linhagens sanguíneas (aplasia medular), podendo consequentemente levar a uma infecção pelo baixo número de células brancas produzidas (8), o que justifica a prevalência na classe "Provável" para Aplasia Medular e Infecção.

Uma das dificuldades encontradas durante a utilização do Algoritmo de Naranjo foi a falta de informações nos prontuários, que consequentemente, levou a uma resposta "desconhecida", não sendo possível pontuar a pergunta ou critério em questão, resultando em uma baixa somatória dos scores.
Tal fragilidade é decorrente da falta de informação quanto aos critérios de retirada e reintrodução do medicamento, à não utilização de placebo, o não relato de ocorrência das mesmas reações anteriormente relatadas, entre outros.

\section{CONCLUSÃO}

O presente estudo traz resultados significativos relacionados à segurança de imunossupressores utilizados em pacientes insuficientes renais internados no hospital universitário em questão. De acordo com o que foi analisado, as principais RAM descritas na literatura e trabalhos semelhantes foram observadas. Diante dos resultados, a ideia de que a presença de um farmacêutico clínico na avaliação farmacoterapêutica dos pacientes é de grande utilidade para o hospital e para a comunidade deve ser reforçada. A atuação do farmacêutico clínico é importante tanto por sua atuação na análise de necessidade, efetividade e segurança dos medicamentos utilizados, quanto pela farmacoeconomia, na busca por menos intercorrências resultantes da farmacoterapia.

\section{REFERÊNCIAS}

1. Kumar V; Abbas AK; Fausto N. Patologia - Bases Patológicas das Doenças. $7^{\mathrm{a}}$ ed. $4^{\mathrm{a}}$ tiragem. Rio de Janeiro: Elsevier. 2005.

2. Riella MC. Princípios de Nefrologia e Distúrbios Hidroeletrolíticos. $5^{\mathrm{a}}$ ed. Rio de Janeiro: Guanabara Koogan. 2010.

3. Smeltzer SC; Bare BG. Tratado de Enfermagem MédicoCirúrgica. $10^{\mathrm{a}}$ ed. Rio de Janeiro: Guanabara Koogan. 2005.

4. SBN. Sociedade Brasileira de Nefrologia. Censo de diálise revela 40 mil novos pacientes em 2017 no Paraná. SBN Informa. 2018;(114):18-22

5. Rang HP; Ritter JM; Flower RJ; Hendernson G. Farmacologia. 8 ed. Rio de Janeiro: Elsevier. 2016.

6. Atala A. Regenerative medicine strategies. J Pediatr Surg. 2012;47(1):17-28. DOI: 10.1016/j.jpedsurg. 2011.10.013

7. Silva P. Farmacologia. $8^{\mathrm{a}}$ ed. Rio de Janeiro: Guanabara Koogan. 2010.
8. Tizo JM, Macedo LC. Principais complicações e efeitos colaterais pós-transplante renal. Rev Unin Rev. 2015;24(1): 62-70

9. KalluriHV; Hardinger KL. Current state of renal transplant immunosuppression: Present and future. World $\mathrm{J}$ Transplant. 2012;2(4):51-68. DOI: 10.5500/wjt.v2.i4.51

10 Fireman MJ; DiMartini AF; Armstrong SC; Cozza KL. Immunosuppressants. Psychosomatics. 2004;45(4):354360. DOI: $10.1176 /$ appi.psy.45.4.354

11 Ferracini FT; Almeida SM; Locatelli J; Petriccione S; Haga CS. Implantação e evolução da farmácia clínica no uso racional de medicamentos em hospital terciário de grande porte. Einstein. 2011;9(4):456-60.

12. Naranjo CA; Busto U; Sellers EM; Sandor P; Ruiz I; Roberts EA; Janecek E; Domecq C; Greenblett DJ. A method for estimating the probability of adverse drug reactions. Clin. Pharmacol. Ther., 1981. 30(2):239-245

13. Capucho HC. Farmacovigilância hospitalar: processos investigativos em farmacovigilância. Farmácia Hospitalar [Encarte]. Brasília. Rev Pharm Bras. 2008(67); 1-12 
14. Cherchiglia ML; Machado EL; Szuster DAC; Andrade EIG; Acúrcio FA; Caiaffa WT; Sesso R; Guerra Jr AA; Queiroz OV; Gomes IC. Perfil epidemiológico dos pacientes em terapia renal substitutiva no Brasil, 2000-2004. Rev Saúde Pública. 2010;44(4):639-649. DOI: $10.1590 /$ S0034-89102010000400007.

15. Pedro JCDA. Transplantação renal no idoso. [Artigo de Revisão]. Universidade de Coimbra, Faculdade de Medicina, 2015.

16. Ajzen H; Schor N. Guias de medicina ambulatorial e hospitalar: Nefrologia. 2a ed. Barueri: Manole. 2005. p. 301-11.

17. Vitko S; Vitko S; Wlodarczyk Z; Kyllönen L; Czajkowski Z; Margreiter R; Backman L; Perner F; Rigotti P; Jaques B; Abramowicz D; Kessler M, Sanchez-Plumed J; Rostaing L; Rodger R S; Donati D; Vanrenterghem Y. Tacrolimus Combined with Two Different Dosages Of Sirolimus in Kidney Transplantation: Results of a Multicenter Study. Am J Transplant 2006;6(3):531-538. DOI: $10.1111 / \mathrm{j} .1600-6143.2005 .01193 . \mathrm{x}$

18. Korolkovas A. Dicionário Terapêutico Guanabara. Ed 2013/2014. Rio de Janeiro: Guanabara Koogan. 2013.
19. Haddad L; Andrade K; Mendes L; Ducatti L; D'Albuquerque LA; Andraus W. Association Between Readmission After Liver Transplant and Adverse Immunosuppressant Reactions: A Prospective Cohort With a 1-Year Follow-up. Transplant Proc. 2017;49(2): 330-337. DOI: 10.1016/j.transproceed.2016.12.005

20. Bucharles SGE; Pecoits-Filho R. Doença Renal Crônica: Mecanismos da Progressão e Abordagem Terapêutica. J Bras Nefrol. 2009;31(1):6-12.

21. Riggs JE. Neurologic Manifestations of Electrolyte Disturbances. Neurol Clin. 2002;20(1):227-239. DOI: 10.1016/S0733-8619(03)00060-4

22. Manfro RC. Manejo da doença crônica do enxerto renal. J Bras Nefrol. 2011;33(4):485-92.

23. Gnatta D. Uso de tacrolimo no desenvolvimento de diabete melito pós-transplante renal. [Dissertação]. Porto Alegre: Faculdade de Farmácia, Universidade Federal do Rio Grande do Sul. 2009.

24. Conrad AT. Análise do custo econômico do transplante renal. [Dissertação]. Porto Alegre: Pontifícia Universidade Católica do Rio Grande do Sul. 2014. 